\title{
Klebsiella pneumoniae Nosocomial Infection in an African Pediatrics Health Center: Case of Campus-Teaching Hospital in Togo
}

\author{
Nadiedjoa Kokou Doutia , Mawouto Fiawoo ${ }^{\text {b, f }}$, Mounerou Salouc ${ }^{c}$ Kossi Mawududzi Senagbe ${ }^{\text {, }}$ \\ Manani Hemou e, Essi Ememe Sanni ${ }^{\mathrm{e}}$, Dzayisse Yawo Atakouma ${ }^{\mathrm{b}}$, \\ Bakoe Bakonde ${ }^{a}$, Mireille Prince-David ${ }^{\mathrm{c}}$
}

\begin{abstract}
Background: The target of our study was to analyze clinical, paraclinical and environmental aspects of a nosocomial infection that occurred at the pediatrics division at Campus Teaching Hospital.

Methods: This was a prospective study initiated after observation of some suspicious cases of nosocomial infection amongst patients. Forty-one children from 29 days to 15 years of age admitted to the Pediatrics Division at Campus Teaching Hospital for a period of 3 months (from April 1 to June 31, 2016) were taken into account in this study. In our study, we included all sick children who showed a reappearance of severe infectious syndrome after 3 days of admission and whose blood cultures were positive. During this period of study, 20 nurses and six medical doctors were questioned as regards to hands clean during the discharge of their work. Studied parameters were: age, sex, initial treatment, bacteria found, sensibility of bacteria, swab results on medical materials in use and hands care.

Results: During the study, 41 cases of blood cultures were identified, out of which $30(73.17 \%)$ tested positive to Klebsiella pneumoniae. The most concerned range of age was between 1 and $5(56.09 \%)$. The initial diagnoses were dominated by 27 malaria $(65.85 \%)$ and gastroenteritis fever (17.08\%). All isolated cases of Klebsiella pneumoniae in our study were multi-resistant but sensitive to colistin. Two children out of 30 infected by Klebsiella pneumoniae died. During the period of study, health staff on duty did not regularly practise proper hands hygiene.
\end{abstract}

Manuscript submitted September 1, 2018, accepted September 25, 2018

aDepartment of Pediatrics, Campus Teaching Hospital of Lome, Lome, Togo bDepartment of Pediatrics, Sylvanus Olympio Teaching Hospital of Lome, Lome, Togo

'Department of Bacteriology and Virology, Sylvanus Olympio Teaching Hospital of Lome, Lome, Togo

${ }^{\mathrm{d} U T ~ S o u t h w e s t e r n ~ M e d i c a l ~ C e n t e r, ~ D a l l a s, ~ T X, ~ U S A ~}$

${ }^{\mathrm{e} C}$ ampus Teaching Hospital of Lome, Lome, Togo

${ }^{f}$ Corresponding Author: Mawouto Fiawoo, Department of Pediatrics, Sylvanus Olympio Teaching Hospital of Lome, Lome, Togo.

Email: michaelisfirst@yahoo.fr

doi: https://doi.org/10.14740/ijcp313w
Conclusions: The case of nosocomial infection is very common in African health centers and it should draw the attention of health officials.

Keywords: Nosocomial infection; Children; Togo

\section{Introduction}

Nosocomial infections constitute a real major public health problem as regards to their frequent crescent, their gravity with regard to the multi resistance in bacteria involved. It can implicate either a patient who has been admitted or got ambulatory treatments or by medical personnel in the course of his professional duties [1]. Contrary to developed countries, little data are available in developing countries especially in sub-Saharan Africa [2]. The purpose of our study is to analyze the clinical, para-clinical and environmental aspects of a nosocomial infection occurred at the Pediatrics Division at the Campus Teaching Hospital.

\section{Materials and Methods}

It was a prospective study initiated after observation of some suspect cases (reappearance of infectious syndrome after 3 days of admission, characterized by a high temperature: 40 - $40.5{ }^{\circ} \mathrm{C}$ with shivers and sometimes with vomiting). The study took place at the Pediatrics Division of Campus Teaching Hospital of Lome. This division has a capacity of 34 beds and admits about 1,400 patients per year. Forty-one children from 29 days to 15 years of age admitted to the Pediatrics Division at Campus Teaching Hospital for a period of 3 months (from April 1 to June 31,2016) were taken into account in this study. In our study, we included all sick children who showed reappearance of severe infectious syndrome after 3 days of admission and whose blood cultures tested positive. During this period of study, 20 nurses and six doctors were questioned on their behavior as regards to hands care during the discharge of their work. Swabs have been done on hospital materials such as beds' mattresses, pillows, portable lamps, drip tubes, bas- 
Table 1. Isolated Bacteria by Blood Culture of the 41 Patients

\begin{tabular}{lcc}
\hline & Case number & Percentage (\%) \\
\hline Escherichia coli & 5 & 12.2 \\
Klebsiella pneumoniae & 30 & 73.2 \\
Salmonella typhi & 3 & 7.3 \\
Staphylococcus aureus & 2 & 4.9 \\
Citrobacter spp & 1 & 2.4 \\
Total & 41 & 100 \\
\hline
\end{tabular}

kets, and splints they use while treating these children so as to be submitted to laboratory test.

The blood culture was done on brain heart infusion (BHI) medium, which is useful for cultivating a wide variety of microorganisms (aerobic, anaerobic and yeast). After cleaning the skin by using polyvinylpyrrolidone or alcohol $70 \%$ as antiseptic, with a syringe, $5 \mathrm{~mL}$ of venous blood was collected. The blood was introduced into a sterilized bottle of $100 \mathrm{~mL}$ of BHI. Thus, the sample obtained is put in the oven at $37^{\circ} \mathrm{C}$. Laboratory technician carried out a visual inspection of the bottle every day to detect a sign of positivity. Bacteria isolation and identification were operated manually based on Gram stain and culture in specific media on agar plate. The laboratory does not perform anaerobic bacteria isolation. Antimicrobial susceptibility testing (AST) was performed on Muller Hinton agar plate. The interpretation of the AST was based on the 2015 CASFM/EUCAST criteria [3].

Studied parameters were: age, sex, initial treatment, bacteria in study, bacteria sensitiveness, swabs' results on medical materials used and hands clean.

\section{Results}

We registered in a total of 41 positive cases of blood cultures in 3 months. Male predominance was observed (26 boys and
15 girls). The most concerned range of age was between 1 to 5 years old in 23 cases $(56.09 \%)$, followed by those of 29 days to 12 months in 10 cases $(24.39 \%)$. Initial diagnose of malaria was made in 27 patients $(65.85 \%)$, gastroenteritis with dehydration $(17.08 \%)$, two cases of pneumopathy $(4.87 \%)$. Drugs used to start the treatment were quinine $(87.12 \%)$, netilmicin $(53.33 \%)$, the third generation cephalosporin (43.13\%), amoxicillin (23.33\%), artemether (6.67\%), ciprofloxacin $(6.57 \%)$, association of acid clavulanic amoxicillin (3.33\%). Initial evolution (in 41 patients) $48 \mathrm{~h}$ after the beginning of the initial treatment was favorable in 40 children $(97.56 \%)$. Regarding blood culture we have noticed Klebsiella pneumoniae predominance with patients: 30 cases out of 41 found bacteria (73.17\%) (Table 1). Cases of isolated Klebsiella pneumoniae by blood cultures were sensitive to colistin only and practically resistant to other antibiotics. Treatment was conditioned by the sensitiveness of the bacteria; all these infected children (about 100\%) by Klebsiella pneumoniae (30 children) were treated with an effective injection of colistin (Table 2). The evolution of infected patients by Klebsiella pneumoniae in our study was favorable in 28 patients $(93.33 \%)$. We have nevertheless registered two cases $(6.67 \%)$ of death. Each room was equipped with 10 beds and one bathroom each. People using this bathroom were more than 10 (sanitary facilities was used by patients as well as by their care takers and hospital staff (nurses, doctors, home nurse). The number of washbasins per room was 2 and was used by patients care takers and hospital staff (doctors, nurses and home nurses). These washbasins facilities were irregularly operational due to water cuts from time to time in these rooms. As for hands care, the six doctors present in the room during the study said that they had irregularly disinfected their hands after the examination of each patient and they had no hydro-alcoholic solution to sanitize their hands. Other workers of the like were 20 in number. Seventeen over $20(85 \%)$ confirmed to be used to washing their hands only when they have finished taking care of the patients and not after each patient examination. Three nurses (15\%) specified doing it irregularly as they finish the treatment.

Table 2. Sensibility of Isolated Bacteria

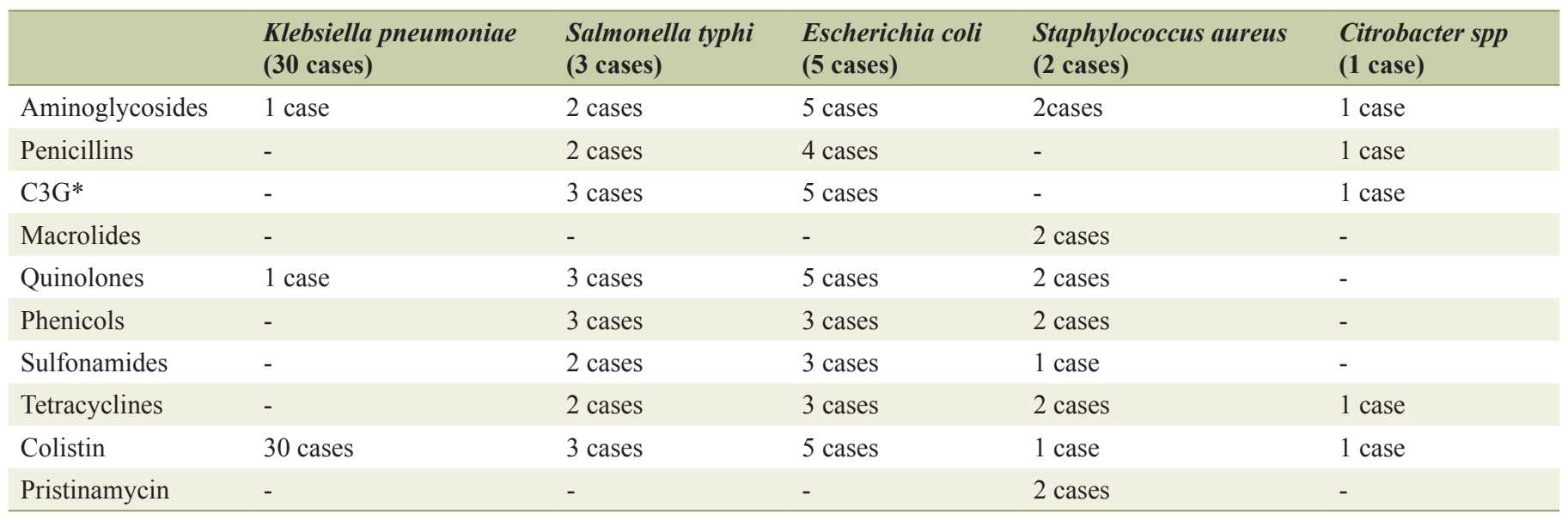

*The third generation cephalosporin. 


\section{Discussion}

Our study has been carried out in sub-Saharan Africa where there is a crucial need of current materials and the precariousness hospital hygiene and sometimes associated with religious and traditional belief do not enable us to deal with nosocomial infections. We wanted through this specific case in Togo to draw the attention of medical personnel on the existence of nosocomial infection in our health centers.

Our study revealed that $73.17 \%$ of bacteria were Klebsiella pneumoniae, and the number of infected males was predominant. This predominance was highlighted in our study $[2,4]$. The most affected age in our study ranged between 12 months to 5 years followed by those of 29 days to 12 months. According to the study of Hugo Sax et al [5] in Switzerland, the frequency of pediatrics nosocomial infections is conditioned by the age of the child. Regarding the results of the studies of these authors, the number of the disease is higher in children under 24 months $(11.5 \%)$ and later on decreased $(3.6 \%)$ in children ranging between 2 to 4 years and $2.6 \%$ in those above 5 years. In our study, the predominance of the infection in children ranging between 12 months to 5 years relatively to those in 29 days to 12 months was due to a bias selection against our will because we noticed in overall a predominance of children of this range of age amongst admitted children during our study. Admitted patients so to say in our study with heavy pathologies at intensive care units were the most vulnerable to nosocomial infections [5]. However, most of patients were admitted for malaria and hospitalized at ordinary structure. The high frequency of Klebsiella pneumoniae in nosocomial infections was highlighted in our study [6, 7]. The isolated Klebsiella pneumoniae from our patients shows similar sensitivity as the ones isolated from materials and tools at the hospital. It was multi-resistant to antibiotics but sensible to colistin. The resistance of enterobacteria to antibiotics particularly Klebsiella pneumonia, was demonstrated in 2010 by Gregory et al [8] in Pueto Rico. This resistance of Klebsiella pneumonia to different antibiotics was also studied by Petrovici et al in Roumania in 2011 [9].

All infected children by Klebsiella pneumoniae were treated through an effective injection of colistin. The fact that colistin was in upsetting in Togo, the medical staff decided to order it for the treatment of these patients. Both cases of death registered probably resulted in the delay caused by the reception of colistin. In West Africa, as well as in most countries with limited health services resources, we do not have sufficient qualified personnel in the field of infectiology, microbiology or epidemiology to set a precise diagnose, suggesting an adapted treatment [10]. Anarchic prescription of antibiotics by any non-qualified medical worker and the easiness to get these antibiotics in the pharmacy contribute to the emergence of resistance of bacteria to antibiotics. Both Ouedraogo in Burkina Faso [10] and Afle [11] in Benin signaled in 2017 the emergence of multi-resistant Klebsiella pneumoniae nosocomial infections in West Africa.

Nosocomial infection depends on several factors. In our study, we analyzed our workplace and noticed that there is one sanitary unit per 10 patients. Since patients come along with families, the total number of people per sanitary unit is more or equal to 20. This situation causes the presence and the development of nosocomial bacteria and infections [12]. In addition, the medical personnel that take care of these patients asserted that to be not used to regularly washing their hands while moving from one patient to another. This situation has also shown that medical personnel lack gel sanitizer in the service. Scheithauer et al in his study demonstrated the importance of hand hygiene [13].

In a study conducted at New York, Son [14] specified that apart from the main measures of hands hygiene globally recommended, simple hands hygiene practices should be encouraged (hands washing and the use of sanitizing gels) which enable medical personnel to always keep hands clean. Kalenic et al [15] at Zagreb suggest that hands clean must be the first concern of government in all medical professional services so as to reduce nosocomial infections.

\section{Conclusions}

Nosocomial infection due to Klebsiella pneumoniae is frequent and can be deadly if neglected. The absence of adequate medical techniques, personnel, poor sanitary measures contribute to spray gems, increase infections and patient death rate.

\section{Conflict of Interest}

The authors declare no conflict of interest.

\section{References}

1. Al-Hajje A, Ezedine M, Hammoud H, Awada S, Rachidi S, Zein S, Salameh P. [Current status of nosocomial infections in the Lebanese Hospital Center, Beirut]. East Mediterr Health J. 2012;18(5):495-500.

2. Saleem M, Vaish AK, Idriss MZ, Sonkar AA, Agarwa J, Singh M, et al. Pattern of nosocomial infection among patients admitted in medical and surgical wards of a secondary care hospital in north India. An epidemiological evaluation. Indian journal of community health, 2012;4(4):285-290.

3. Jehl F, Bonnet R, Bru J-P, Caron F, Cattoir V, Chardon H et al. Comite de l'antibiogramme de la societe francaise de microbiologie. Recommandations 2015 V.2.0 Juillet. Available at: http://www.sfm-microbiologie.org/UserFiles/ files/casfm/CASFMV2_030915.pdf Accessed 22 September 2018.

4. Togo A, Coulibaly Y, Keita M, Traore A, Kante L, Diakite I, et al. Infections nosocomiales en chirurgie pediatrique au Mali. Journal de pediatrie et de puericulture. 2009;22:273-277.

5. Hugo S, Didier P. Infections nosocomiales et hygiene hospitaliere: Aspects actuels. Resultats de l'enquete nationale de prevalence des infections nosocomiales de 2004 (snip04). Swiss-NOSO 2005;12(1):1-8. 
6. Compaore TSA, Gueye Ba M, Ka AS, Dionne P, Wade B. Surveillance des infections nosocomiales: bilan de quatre annees d'enquete de prevalence « un jour donne » dans le departement Mere-Enfant de l'hopital Principal de Dakar (Senegal). Revue de medecine perinatale. 2010;2:213218.

7. Carbonne A, Thiolet JM, Fournier S, Fortineau N, KassisChikhani N, Boytchev I, Aggoune M, et al. Control of a multi-hospital outbreak of KPC-producing Klebsiella pneumoniae type 2 in France, September to October 2009. Euro Surveill. 2010;15(48).

8. Gregory CJ, Llata E, Stine N, Gould C, Santiago LM, Vazquez GJ, Robledo IE, et al. Outbreak of carbapenemresistant Klebsiella pneumoniae in Puerto Rico associated with a novel carbapenemase variant. Infect Control Hosp Epidemiol. 2010;31(5):476-484.

9. Petrovici CG, Dorobat C, Matei M, Teodor A, Luca V, Miftode E. [Aspects of the antimicrobial resistence profile in infections with Escherichia coli and Klebsiella pneumoniae in diabetic patients]. Rev Med Chir Soc Med Nat Iasi. 2011;115(3):769-775.

10. Ouedraogo AS, Jean Pierre H, Banuls AL, Ouedraogo R, Godreuil S. Emergence et diffusion de la resistance aux antibiotiques en Afrique de l'Ouest: facteurs favorisants et evaluation de la menace. Medecine et Sante Tropicales. 2017;27:147-154.

11. Afle FCD, Quenum KJMK, Hessou S, Johnson RC. Etat des lieux des infections associees aux soins dans deux hopitaux publics du sud Benin (Afrique de l'ouest): Centre Hospitalier Universitaire de Zone d'Abomey Calavi/SoAva et Centre Hospitalier de Zone de Cotonou 5. Journal of Applied Biosciences. 2018;121:12192-12201.

12. Chung HC, Lee RP. [Multidrug-resistant bacteria in the hospital: the focus of nursing care]. $\mathrm{Hu} \mathrm{Li} \mathrm{Za} \mathrm{Zhi.}$ 2011;58(4):11-15.

13. Scheithauer S, Schwanz T, Lemmen S. [Hand hygiene: basic, but not trivial]. Laryngorhinootologie. 2011;90(7):434-443; quiz 444-436.

14. Son C, Chuck T, Childers T, Usiak S, Dowling M, Andiel $\mathrm{C}$, Backer R, et al. Practically speaking: rethinking hand hygiene improvement programs in health care settings. Am J Infect Control. 2011;39(9):716-724.

15. Kalenic S, Budimir A, Bosnjak Z, Acketa L, Belina D, Benko I, Bosnjak D, et al. [Guidelines on hand hygiene in health care institutions]. Lijec Vjesn. 2011;133(5-6):155170 . 